\title{
Impact of Respiratory Fluctuation on Hemodynamics in Human Cardiovascular System: A 0-1D Multiscale Model
}

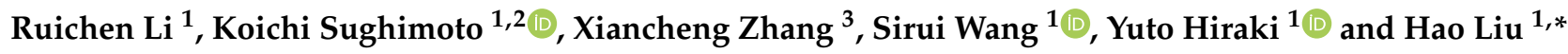 \\ 1 Faculty of Engineering, Chiba University, Chiba 263-8522, Japan; liruichen0213@chiba-u.jp (R.L.); \\ ksughimoto@gmail.com (K.S.); siruiwang615@outlook.com (S.W.); yutohiraki@chiba-u.jp (Y.H.) \\ 2 Chiba Kaihin Municipal Hospital, Chiba 261-0012, Japan \\ 3 Shenzhen Institute of Advanced Technology, Chinese Academy of Sciences, Shenzhen 518055, China; \\ xc.zhang@siat.ac.cn \\ * Correspondence: hliu@faculty.chiba-u.jp; Tel.: +81-(0)43-290-3228
}

Citation: Li, R.; Sughimoto, K.; Zhang, X.; Wang, S.; Hiraki, Y.; Liu, H. Impact of Respiratory Fluctuation on Hemodynamics in Human

Cardiovascular System: A 0-1D

Multiscale Model. Fluids 2022, 7, 28

https://doi.org/10.3390/

fluids7010028

Academic Editor: Mehrdad Massoudi

Received: 1 December 2021

Accepted: 5 January 2022

Published: 7 January 2022

Publisher's Note: MDPI stays neutral with regard to jurisdictional claims in published maps and institutional affiliations.

Copyright: (C) 2022 by the authors. Licensee MDPI, Basel, Switzerland. This article is an open access article distributed under the terms and conditions of the Creative Commons Attribution (CC BY) license (https:// creativecommons.org/licenses/by/ $4.0 /)$.

\begin{abstract}
To explore hemodynamic interaction between the human respiratory system (RS) and cardiovascular system (CVS), here we propose an integrated computational model to predict the CVS hemodynamics with consideration of the respiratory fluctuation (RF). A submodule of the intrathoracic pressure (ITP) adjustment is developed and incorporated in a 0-1D multiscale hemodynamic model of the CVS specified for infant, adolescent, and adult individuals. The model is verified to enable reasonable estimation of the blood pressure waveforms accounting for the RF-induced pressure fluctuations in comparison with clinical data. The results show that the negative ITP caused by respiration increases the blood flow rates in superior and inferior vena cavae; the deep breathing improves the venous return in adolescents but has less influence on infants. It is found that a marked reduction in ITP under pathological conditions can excessively increase the flow rates in cavae independent of the individual ages, which may cause the hemodynamic instability and hence increase the risk of heart failure. Our results indicate that the present 0-1D multiscale CVS model incorporated with the RF effect is capable of providing a useful and effective tool to explore the physiological and pathological mechanisms in association with cardiopulmonary interactions and their clinical applications.
\end{abstract}

Keywords: cardiovascular system; respiratory fluctuation; 0-1D multiscale hemodynamic model; intrathoracic pressure; cardiopulmonary interactions

\section{Introduction}

With continuous and remarkable progress of life science and biomedical technology in the past few decades, both theoretical and clinical practice research on multiple interactions between various systems of living organisms, particularly between the respiratory system and cardiovascular system (CVS), has attracted increasing attention because of its noticeable influence on individual human health. Clinical measurements [1] show that blood pressure (BP) waveforms present longer periodic fluctuations during the cardiac cycle with breathing, and its fluctuation frequency is highly dependent upon respiratory frequency while its fluctuation amplitude increases with decreasing respiratory rate. Previous studies [2-7] have reported that the negative value of intrathoracic pressure (ITP) caused by inhalation can promote more blood pooling into the heart through superior and inferior vena cavae while reducing blood resistance at the peripheral vascular level of major organs, thus enhancing ventricular filling while increasing stroke volume or even resulting in improvement of tissue perfusion. Efforts have also been made to improve the CVS hemodynamics by means of in vivo methods to regulate spontaneous breathing or ventilation reasonably while enhancing cardiopulmonary function for healthy people and patients with cardiovascular diseases (CVDs), particularly to help treat significant, often lifethreatening, circulatory disorders [8]. Although the respiratory system can play a crucial 
role in altering the hemodynamics in human CVS, a comprehensive understanding of the impact of respiratory fluctuation on the CVS hemodynamics remains poorly understood.

The anatomical complexity and nonlinear dynamics of the human CVS have added significant obstacles to clinical trials and sophisticated 3-dimensional (3D) computational fluid dynamic (CFD) modeling as well as individual-specific hemodynamic analysis. The reduced order, 0-1-dimensional (0-1D) modeling of CVS hemodynamics that benefits both computer time consumption and resources compared with a fully 3-dimensional CFD-based simulation, has been widely recognized as a versatile and effective tool to examine both physiological and pathological CVS hemodynamics, and to explore clinical applications [9-12]. The 0D lumped-parameter models based on the Windkessel model and the principles of electrical analog, coupling with 1D mathematical models for blood flow and pulse wave propagation [13-16], have been adopted to describe the hematological characteristics of arterial entrance and arterial-venous interface through defining parameters such as vascular elasticity and blood flow resistance [17]. Formaggia et al. [18] proposed a simple lumped-parameter model of the heart (left ventricle), which is coupled with a 1D arterial model to investigate the interaction of the heart arteries. Liang et al. $[19,20]$ developed a 0-1D multiscale CVS model for the heart and arterial-tree while applying it to assess the clinical measurement diagnostic process [21-23] and the treatment of CVDs including the analysis of long-term performances and transient variations in the CVS hemodynamics during the perioperative of carotid artery surgery and Fontan circulation [24-27]. Müller et al. [28] expanded the 0-1D modeling methodology to involve the heart, the arterial-tree and the arteriovenous system, the so called global multiscale mathematical model for the human CVS. More recently, Zhang et al. [29,30] established a close-loop CVS model for the systematic circulation, enveloping the heart, arterial system and the venous system, being coupled with a $0 \mathrm{D}$ lump-parameter model at the capillary interface between arterial and venous systems, while accounting for the gravitational effect and aging-specification on the CVS hemodynamics.

While the respiratory regulation is considered beneficial to improve the characteristics of central hemodynamics in the CVS, it remains poorly studied in association with the respiratory fluctuation (RF) effects on the CVS hemodynamics, particularly by means of the 0-1D multiscale hemodynamic modeling. This study aims to establish a clinically useful and effective 0-1D CVS model with consideration of the RF effect and unraveling the RF impact on human CVS hemodynamics. The model is capable of examining the effects of various breathing patterns on both systemic and local hemodynamics while providing simulation-driven useful guidance for personalized respiratory-based health management and disease treatment options for healthy people or CVD patients.

\section{Materials and Methods}

An integrated computational model that incorporates the RF effect into the 0-1D multiscale hemodynamic modeling of the CVS hemodynamics is developed to explore the RF-induced impact on blood pressure and flow rates in association with cardiopulmonary interactions. As illustrated in Figure 1, the RF waveforms that are digitized based on clinical measurements are expressed in a manner of Fourier series and incorporated into an in-house 0-1D hemodynamic model. This is further implemented by replacing the conventional constant and negative ITP $[8,30]$ in the equation of blood pressure with a time varying ITP-related contribution term so that the impact of respiration-induced RF on CVS hemodynamics can be predicted effectively. Furthermore, considering that the data collected in the intensive care unit (ICU) contained a certain number of underage patients, we herein introduced an age adjustment mechanism into the 0-1D hemodynamic model on the basis of the allometric scaling law (ASL) [31,32]. 


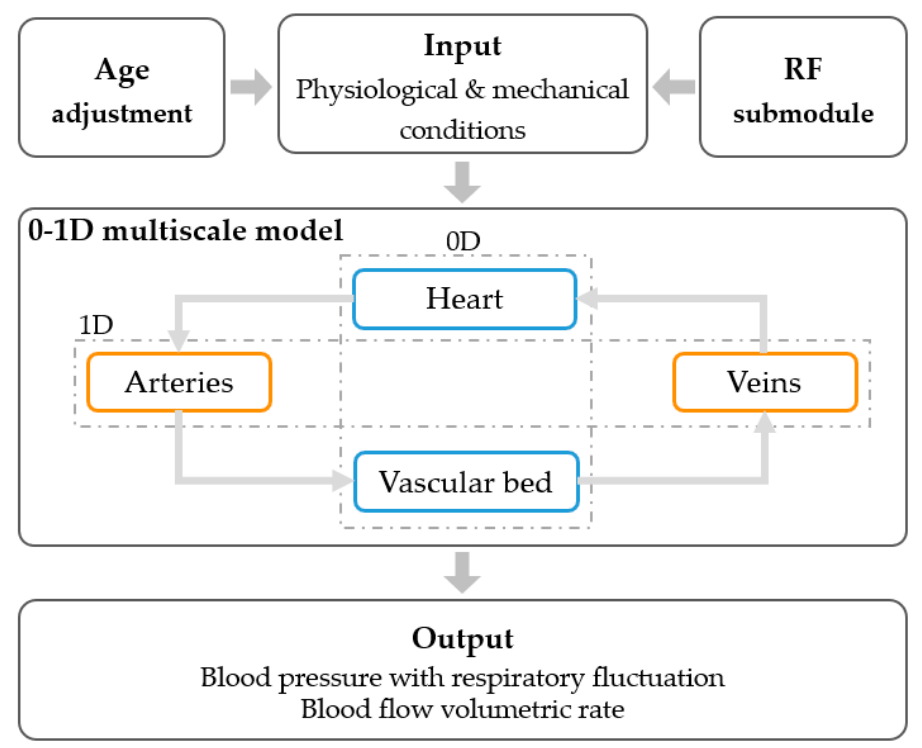

Figure 1. Flowchart of the 0-1D multiscale hemodynamic modeling with consideration of RF. The physiological and mechanical conditions associated with RF effect and age adjustment are incorporated into a 0-1D multiscale model.

\subsection{0-1D Modeling of Hemodynamics in Human CVS}

The newly developed 0-1D multi-scale hemodynamic model [9,30] is given in a closed loop manner, which enables us to reasonably calculate both blood pressure and flow volumetric rate throughout the entire human CVS. The model is established based on the geometric dimensions and mechanical properties of population-averaged blood vessels identical to a 25-year-old adult weighing $60 \mathrm{~kg}$.

By combining the Windkessel model with Kirchhoff's law, the 0D hemodynamic model is constructed for arterial-venous interface vascular bed and time-varying cardiac function using different elements to represent blood vessel compliance (C) and resistance (R) as well as the blood flow inertance (L) $[19,20,29]$, such as:

$$
\begin{gathered}
\frac{\mathrm{d} V_{i}}{\mathrm{~d} t}=Q_{i-1}-Q_{i}, \\
\frac{\mathrm{d} Q_{i}}{\mathrm{~d} t}=\frac{P_{i}-P_{i+1}-Q_{i} \cdot R_{i}}{L_{i}},
\end{gathered}
$$

where $V_{i}, Q_{i}$ and $P_{i}\left(P_{i}=V_{i} / C_{i}\right)$ denote the blood volume, the flow rate, and the pressure at compartment $i$, respectively.

The governing equations of the 1D hemodynamic model express the conservation of mass and momentum associated with compliant blood vessels with uniform pressure and axial velocity distributions on each axisymmetric cross-section, as well as the constitutive equation that describes the pressure-area relationship of blood vessels $[9,12,30,33]$, which can be derived as:

$$
\begin{gathered}
\frac{\partial A}{\partial t}+\frac{\partial A u}{\partial x}=0, \\
\frac{\partial u}{\partial t}+u \frac{\partial u}{\partial x}+\frac{1}{\rho} \frac{\partial p}{\partial x}=-\xi \frac{\pi \mu}{\rho} \frac{u}{A}, \\
p=\frac{2 \rho c_{0}^{2}}{b}\left[\left(\frac{A}{A_{0}}\right)^{\frac{b}{2}}-1\right]+P_{0}+\text { ITP, }
\end{gathered}
$$

where $A$ and $A_{0}$ denote the cross-sectional area and the reference cross-sectional area, respectively; $p$ and $P_{0}$ represent the average vessel internal pressure over the cross section 
and the reference pressure, respectively; $t$ is the time and $x$ is the axial coordinate; $u$ is the average axial velocity; $\mu, \rho$ and $\xi$ are the blood viscosity, the blood density and viscous friction constant, respectively; $c_{0}$ is the reference pulse wave velocity. The term ITP represents the RF-induced effect and is defined in a manner of time-varying waveforms based on the measurements. Term $b$ is a constant that determines the shape of the pressurearea relationship and is given as:

$$
b=\frac{2 \rho c_{0}^{2}}{P_{0}-P_{\text {collapse }}}
$$

where $P_{\text {collapse }}$ is the collapse pressure and set as $-10 \mathrm{mmHg}$ for all blood vessels.

Here we employed a 1D hemodynamic model comprising 20 major vessels consisting of aortas, vena cavae, pulmonary arteries and veins, with the peripheral blood vessels modeled by a 0D vascular bed model at the intersection of veins and arteries (peripheral bed vessels of systematic circulation and pulmonary circulation), to achieve the closed loop 0-1D multiscale hemodynamic modeling [30].

\subsection{Clinical Data Collection and Processing}

Postoperative arterial pressure waveforms were collected from the patients with CVDs in the intensive care unit (ICU) and the pediatric intensive care unit (PICU). Allowing for the postoperative monitoring system being susceptible to the instability of blood flow caused by many factors from outside (changes in patient posture, process of blood testing on the upper arm, etc.), we chose relatively stable hemodynamics and a comparatively regular period of BP fluctuations for waveform analysis. In addition, since the clinical status of the same patient varies from heartbeat to heartbeat after admission to ICU, we further divided the time course into several different segments in minutes during the periods of the relatively stable hemodynamics. The peak and nadir values of the patients' arterial pressure waveforms, as well as the respiratory-induced fluctuations, are also extracted by alternating the use of high-pass digital filtering and low-pass digital filtering.

Figure 2 shows the representative BP waveforms from the postoperative monitoring system in the PICU ward during the period of one minute of a 12-year-old adolescent patient who underwent ventricular septal defect (VSD) closure. It is observed that, under the condition of a relatively stable state, in addition to the transient BP fluctuations (grey line) associated with heart beats and the irregularly fluctuating waveforms, there apparently exists a featured undulating waveform with a noticeably longer wavelength (red bold line with red inverted triangles pointing to the peaks), which is considered to be caused by the respiratory movements because the fluctuation frequency is consistent with that of human breathing (12-20 cycles per minute).

\subsection{Submodule of Respiratory Fluctuations (RF)}

When the RF waveform in association with the BP waveform (Figure 2) is extracted from the relatively stable state of hemodynamics of patients, the waveform in a complete respiratory cycle is sufficient to approximate the RF waveform within a short period of time, for example, 1-min in this study. Figure 3 shows the comparison results of fitting the RF waveform during a respiratory cycle by using the 1st-order and 2nd-order Fourier series. The R2-scores of both are above 0.98. We found that the RF waveform of patients can be well simulated by using the 1st-order Fourier series. Here, we employ this Fourier series fitting method to approximate the RF waveform based on clinically measured BPs. The time-varying parameter $\operatorname{ITP}(t)$ with RF can be derived by:

$$
\operatorname{ITP}(t)=A_{0}+A_{1} \cos (\omega t)+B_{1} \sin (\omega t),
$$

where $t$ is the time (s), $A_{0}$ is the baseline of ITP with the RF waveform (mmHg), $A_{1}$ and $B_{1}$ are the two coefficients of 1st-order Fourier series, that is, the fluctuating amplitudes of RF $(\mathrm{mmHg})$, and $\omega$ is the respiratory rate during the target period. Combining Equation (7) 
with Equation (5) in the 0-1D multiscale model can thus predict the BP waveform with the RF effect accounted for.

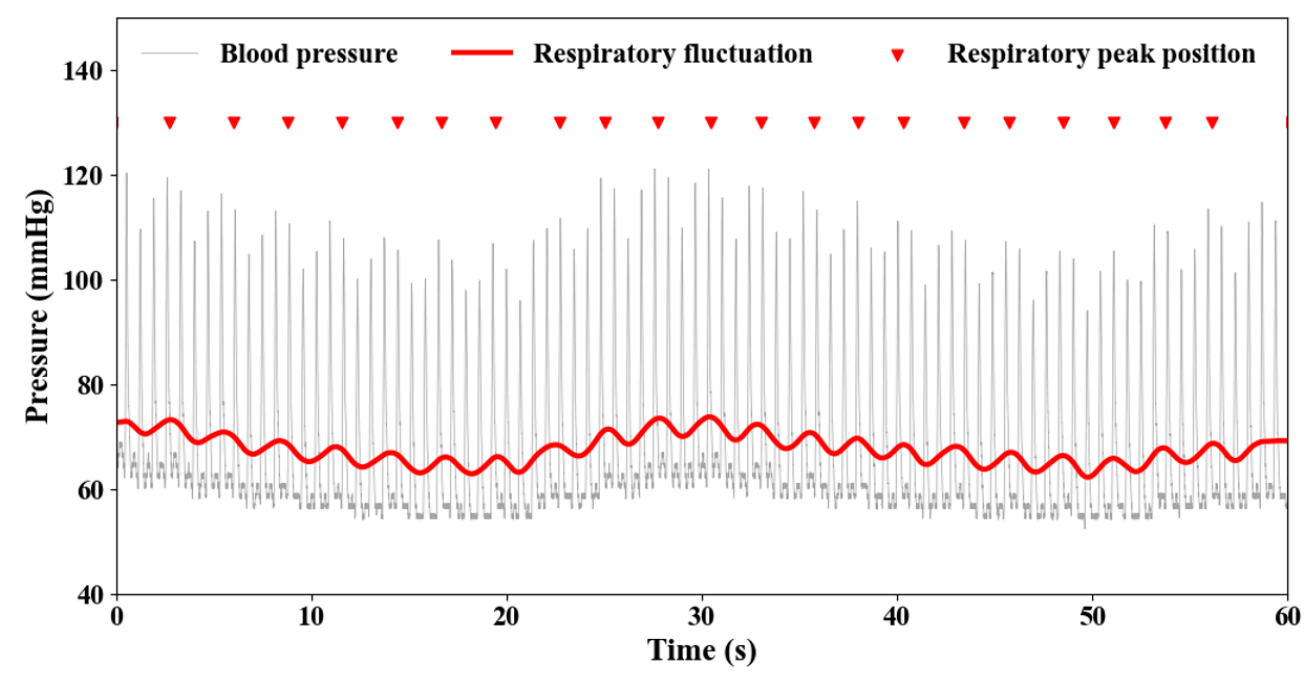

Figure 2. BP and RF waveforms extracted from a 12-year-old VSD patient in $1 \mathrm{~min}$.

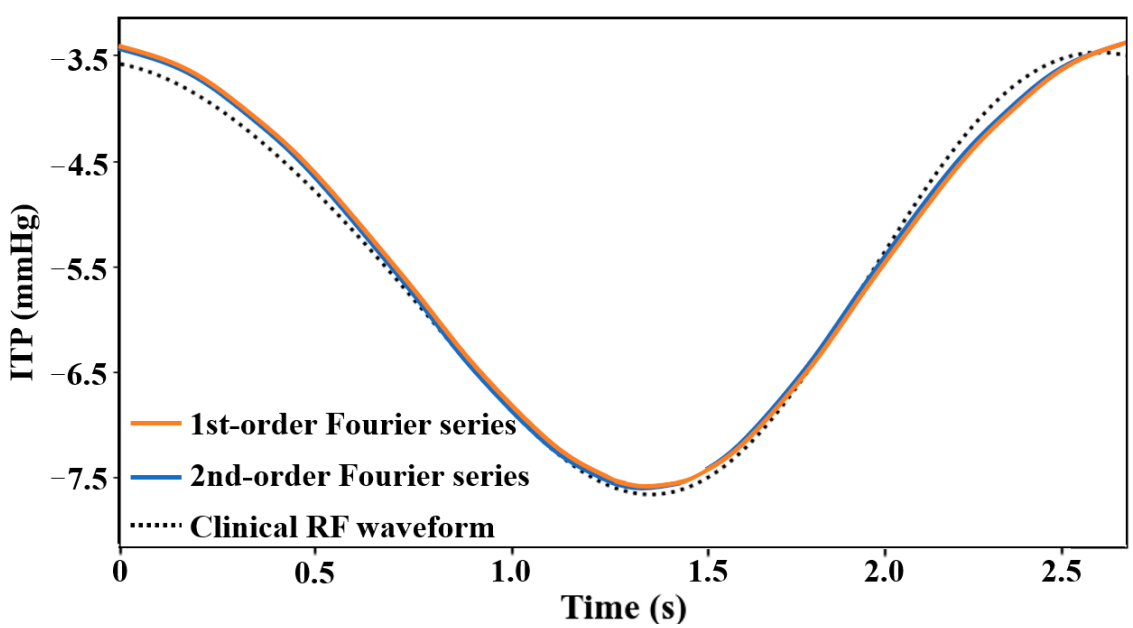

Figure 3. Comparison of fitting RF waveforms in a respiratory cycle between 1st-order and 2nd-order Fourier series approximations.

\subsection{Age Regulation}

Since the clinical data contain a certain number of pediatric patients, it is essential to make appropriate adjustments to the parameters with the consideration of the ages of infants and adolescents. The effect of age regulation, which is employed based on the ASL and incorporated into each submodule in the 0-1D multiscale hemodynamic CVS model, can be indirectly accounted for through adjusting the ratio of the body weight of the target patient or individual to the population-average of an adult aged 20-30 years old and weighing $60 \mathrm{~kg}$ [30].

The age-related cardiovascular parameters are determined by

$$
X=X_{\text {adult }}\left(\frac{W}{W_{\text {adult }}}\right)^{\beta},
$$

where $X$ and $X_{\text {adult }}$ denote various physiological parameters and their population-averaged reference values, respectively; $W$ and $W_{\text {adult }}$ are the weight and reference weight $(60 \mathrm{~kg})$, 
respectively; $\beta$ is a scaling exponent, which varies with various geometric and mechanical parameters of the human CVS, for example, here being set to a value of 0.35 for the case of ITP.

\section{Results and Discussion}

\subsection{RF Modeling Validation}

We first extracted the BP waveforms and their variations of two subjects including a 12-year-old adolescent patient and a 1-year-old infant patient. The specific physiological parameters of the two patients are summarized in Table 1, corresponding to a period when the hemodynamic statues were observed to be relatively stable after admission to the ICU. Since the 0-1D multiscale hemodynamic model employed in this study does not include all the major blood vessels of the CVS, we calculated the BP waveforms of the available brachiocephalic artery. Thus, a comparison of BP waveforms between the simulations in the brachiocephalic artery and the measurement in the radial artery is used to verify the RF submodule in an indirect manner and further investigate the hemodynamic characteristics under the impact of respiration using the modified 0-1D multiscale hemodynamic model with the RF submodule incorporated.

Table 1. Physiological parameters of two research subjects.

\begin{tabular}{ccc}
\hline \multirow{2}{*}{ Types } & \multicolumn{2}{c}{ Parameters } \\
\cline { 2 - 3 } & Subject $\mathbf{~}$ & Subject 2 \\
\hline Age & 12-year-old & 1-year-old \\
Disease & Ventricular septal defect (VSD) & Atrial septal defect (VSD) \\
Weight & $40.8 \mathrm{~kg}$ & $9.3 \mathrm{~kg}$ \\
Heart rate & 86 beats $/ \mathrm{min}$ & $135 \mathrm{beats} / \mathrm{min}$ \\
Cardiac cycle & $0.697 \mathrm{~s}$ & $0.444 \mathrm{~s}$ \\
Respiration & 22 beats $/ \mathrm{min}$ & 24 beats $/ \mathrm{min}$ \\
\hline
\end{tabular}

The simulated results are compared with clinical measurements of the adolescent (12-year-old, VSD) and infant subjects (1-year-old, ASD) as shown in Figures 4 and 5, respectively. In the case of an adolescent subject, the computed RF waveforms show a similar trend with the measurements in terms of respiratory frequency and amplitude but fail to capture the long-wavelength undulation in BP and RF waveforms, which may be due to measurement uncertainties in association with various factors such as unstable external environment and patient-specific difference, and so forth. It is worth noting that the BPs in the brachiocephalic artery predicted by the 0-1D model apparently show a smaller range from systole and diastole (Figure 4) compared to the measurements in the radial artery by approximately $10 \mathrm{mmHg}$. Armstrong et al. [34] also reported that, on the comparison of BPs in brachiocephalic artery and radial artery, most of the participants demonstrated that the radial systolic BP is $5 \mathrm{~mm} \mathrm{Hg}$ higher than the brachial systolic BP, with many even greater than $15 \mathrm{mmHg}$. It is thus verified that the current modified 0-1D multiscale hemodynamic model enables reasonable prediction of the BP waveforms with consideration of the RF effect. Moreover, in the case of an infant subject, as shown in Figure 5a even during the period when the hemodynamic statues are relatively stable, some irregular fluctuations are still observed in the measured BP waveforms, probably due to the measurement uncertainty. Obviously, the simulated BP waveforms (Figure 5b) based on the 0-1D model modified with aging adjustment as described in the preceding section are consistent with the measurements in both the mean value and fluctuation amplitude of the RF waveforms, which are much smaller than those of the adolescents and adults (Figure 4). 


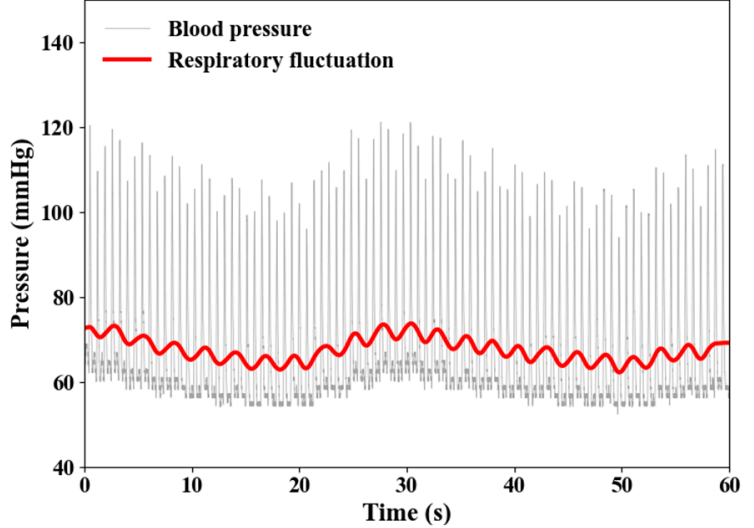

(a)

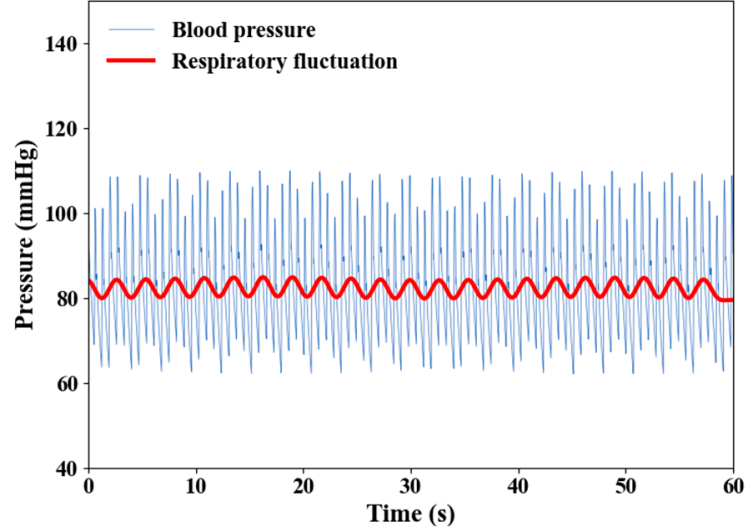

(b)

Figure 4. Comparison of RF waveforms between (a) clinical measurements and (b) 0-1D hemodynamic model-based simulations (subject: 12-year-old, VSD).

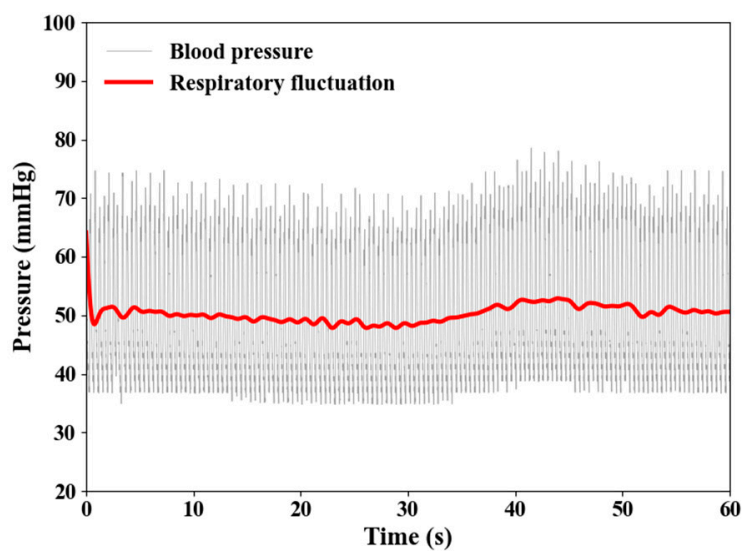

(a)

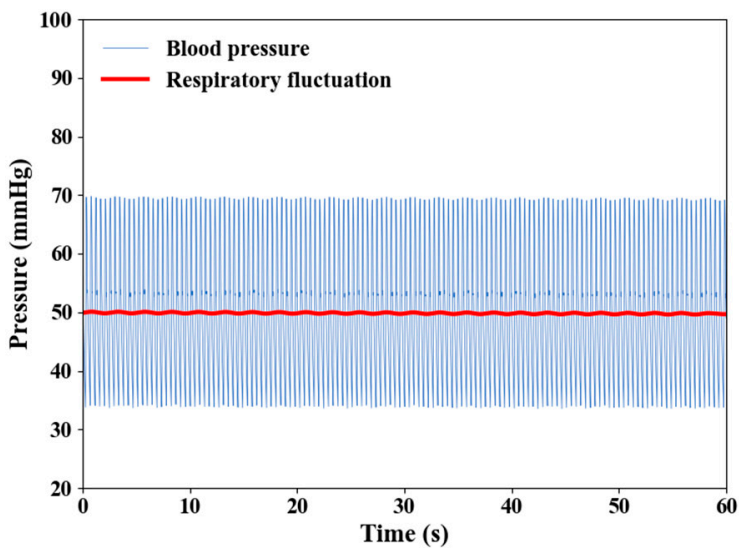

(b)

Figure 5. Comparison of RF waveforms between (a) clinical measurements and (b) 0-1D hemodynamic model-based simulations (subject: 1-year-old, ASD).

\subsection{Impact of RF on Venous Return}

We first applied the 0-1D multiscale hemodynamic model to examine the influence of respiratory exercise on central hemodynamic characteristics with a specific focus on the impact of RF on venous return associated with the superior vena cava (SVC) and inferior vena cava (IVC).

Figure 6 shows the comparison of flow rates and ITPs in the SVC and IVC of an adolescent subject (12-year-old) under the influence of RF in a normal respiratory mode and without the RF effect. The impact of RF on the flow rates in both SVC and IVC (Figure $6 \mathrm{a}, \mathrm{b}$ ) is insignificant but does display some pronounced differences at peak values. During the inhalation, when the ITP shows a graduate decrease to the lower level by a drop of approximately $1.5 \mathrm{mmHg}$, the flow rates at the 2nd and 3rd peaks in SVC and IVC are increased by approximately $5 \mathrm{~mL} / \mathrm{s}$ and $10 \mathrm{~mL} / \mathrm{s}$, respectively, compared to the case without the RF effect. At the end of exhalation, when the ITP returns to the normal level, the flow rates in both cases with/without the RF effect reach a convergent value. 


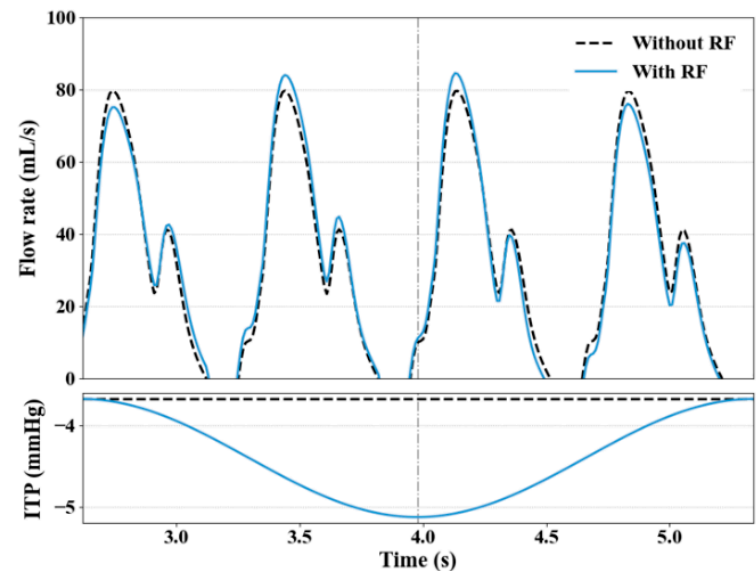

(a)

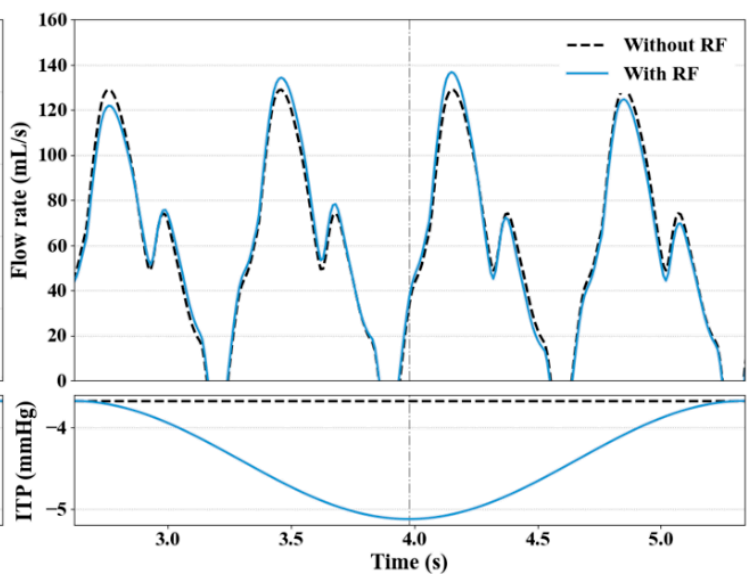

(b)

Figure 6. Flow rates and ITPs in SVC (a) and IVC (b) of an adolescent subject with/without RF effect.

We further investigated the influence of deep respiration on the flow rates in SVC and IVC under two different breathing modes of normal respiration and deep respiration for the adolescent subject with and without the RF influence. Obviously, as illustrated in Figure 7, the deep breathing with an ITP reduction of $4 \mathrm{mmHg}$ enables us to significantly enhance the flow rates, particularly at the end of the inhalation, for instance, at the 2nd and 3rd peaks by a remarkable increase of approximately $20 \mathrm{~mL} / \mathrm{s}$ compared to the normal respiration mode. Again, when the ITP returns to the normal level at the end of exhalating, the flow rates are restored to the same level as in the normal breathing mode independent of the RF impact. This indicates that the deep respiration can effectively improve the hemodynamic characteristics in terms of venous return of the CVS.

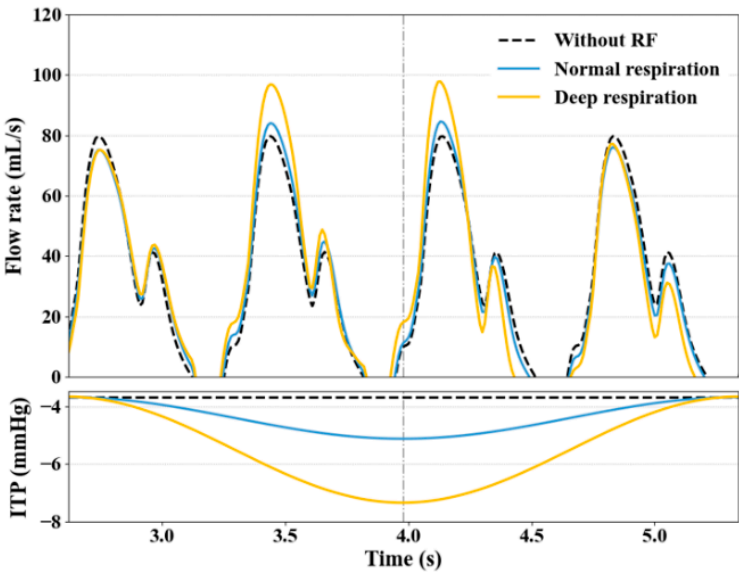

(a)

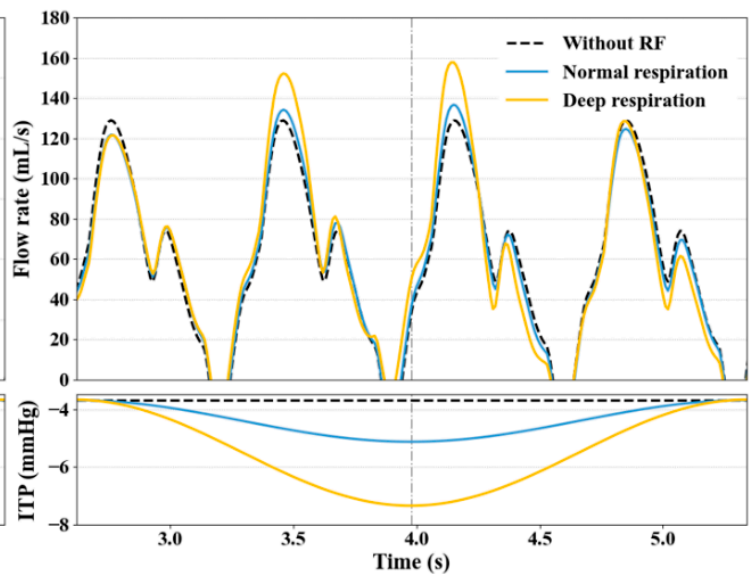

(b)

Figure 7. Flow rates and ITPs in SVC (a) and IVC (b) of an adolescent subject in normal and deep breathing modes and without RF effect.

As indicated in Figures 6 and 7, three key physiological characteristics can be clarified in the waveforms of flow rate in SVC and IVC under the normal and deep respiratory modes in terms of: (1) a large antegrade flow during ventricular systole; (2) a small antegrade flow during ventricular diastole; and (3) a small retrograde flow during right atrial contraction (negative value). These are regarded as the key indicators for judging whether the flow rate varies in SVC and IVC in a normal manner [30,35].

In addition, an extensive study was undertaken on investigating how the breathing mode affects the venous return in an infant (1-year-old) through examining the flow rates in 
SVC and IVC during normal and deep respiration. Unlike the case of the adolescent subject, the difference between normal and deep respiratory modes that correspond to an ITP reduction of $1 \mathrm{mmHg}$ and $1.8 \mathrm{mmHg}$, respectively, results in an insignificant discrepancy in the flow rates in both SVC and IVC (Figure 8). It is worth noting that, given the much smaller body size in infants compared to adolescents, the blood volume and hence flow rates are largely reduced to a lower level. Thus, the breathing mode turns out to bring less impact on the flow rate in both SVC and IVC, and a slight reduction in the peak value is further observed compared to the case without the RF effect at the end of exhalation. This implies that the aging effect may exist in terms of the deep breathing impact on hemodynamic characteristics in association with venous return. Note that the three key physiological features are also confirmed in the flow rate of the infant subject as found in the adolescent and adult subjects.

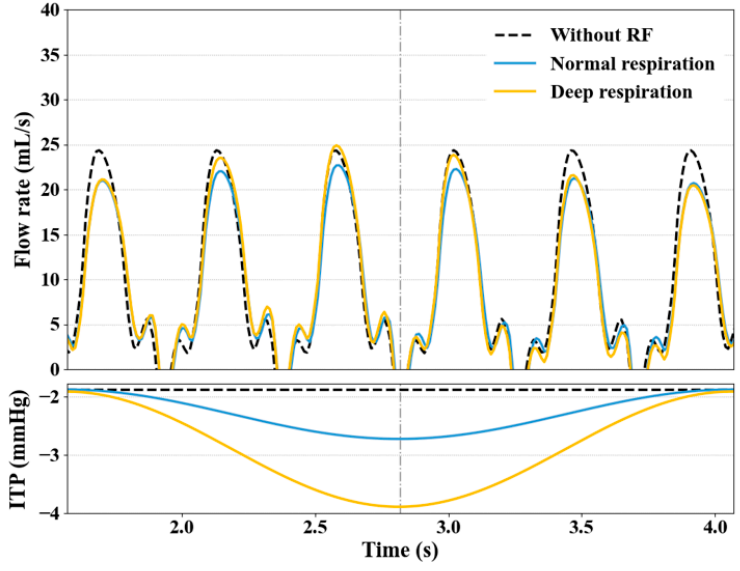

(a)

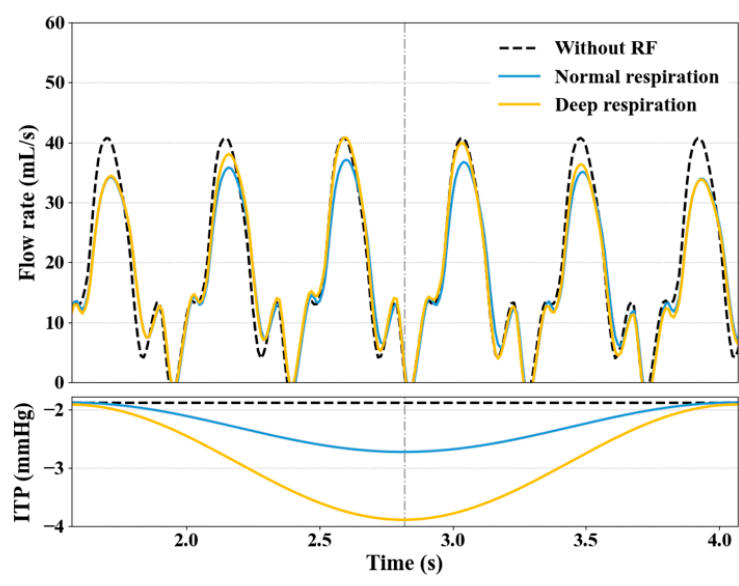

(b)

Figure 8. Flow rates and ITPs in SVC (a) and IVC (b) of an infant subject in normal and deep breathing modes and without RF effect.

\subsection{Effect of Large ITP Reduction}

Apart from the positive influence of reduced ITP fluctuation owing to normal or deep respiration, there are also some different views that a large reduction of ITP can have adverse effects on hemodynamics, for example, leading to limiting venous return [36,37] and reducing cardiac filling [38]. In general, the sharply decreasing ITP during a passive over-deep respiratory cycle is a typical pathological phenomenon, as often observed in patients with basic cardiovascular diseases (CVDs), such as heart failure, chronic obstructive pulmonary disease, asthma and so on.

To investigate such an extreme impact of ITP reduction on hemodynamics, here we further carried out a study to examine how a specific respiratory mode associated with a large reduction can affect the venous return. The reduction range of ITP shown in Figures 9 and 10 was set as $14.7 \mathrm{mmHg}$ (identical to $20 \mathrm{cmH}_{2} \mathrm{O}$ ) [8] and $7.7 \mathrm{mmHg}$ (identical to $10 \mathrm{cmH}_{2} \mathrm{O}$ ) for 12 and 1 year old subjects, respectively. For the 12-year-old subject (Figure 9), at the end of inspiratory period, a small retrograde flow was observed during right atrial contraction at the end of the 2nd cardiac cycle in SVC and IVC, resulting in an irregular waveform in flow rates; at the end of the 3rd cardiac cycle, a marked phase change is present with the reflux in SVC largely advanced and the recovery time of SVC return delayed at the early 3rd cardiac cycle. This phenomenon may cause the instability of venous return, thus substantially degrading the cardiovascular functions. With respect to IVC, on the contrary, the forward flow takes up almost the entire process of the respiratory cycle, indicating that this phenomenon may lead to some excessive preload and hence increasing the risk of heart failure [39]. For the 1-year-old infant subject (Figure 10), the large ITP reduction does not appear to have a substantial impact on the flow rates compared 
to those seen in adolescent and adult subjects, whereas it plays a positive role in enhancing the forward flow in both SVC and IVC, which may also exert some excessively elevated influence on the ventricular preload.

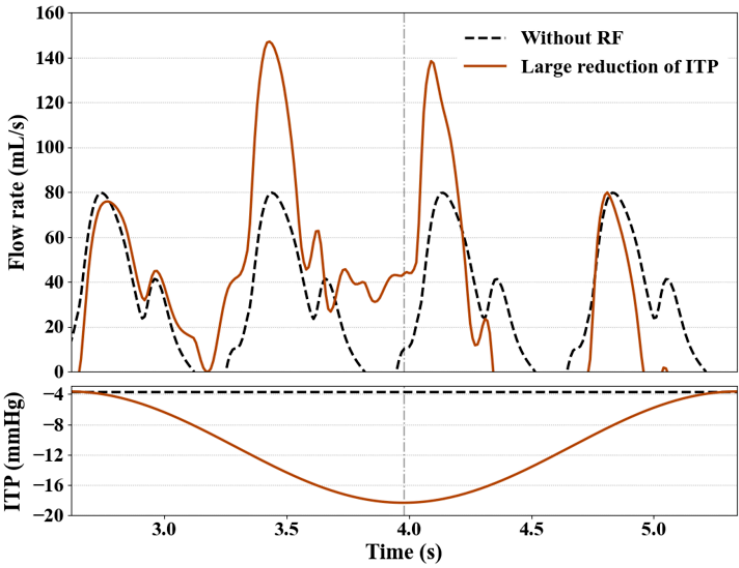

(a)

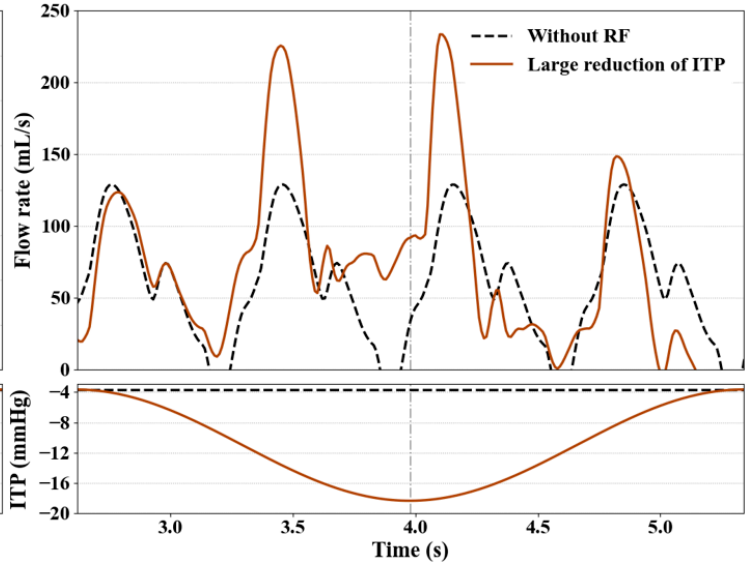

(b)

Figure 9. Impact of large ITP reduction on flow rates in SVC (a) and IVC (b) associated with an adolescent subject.

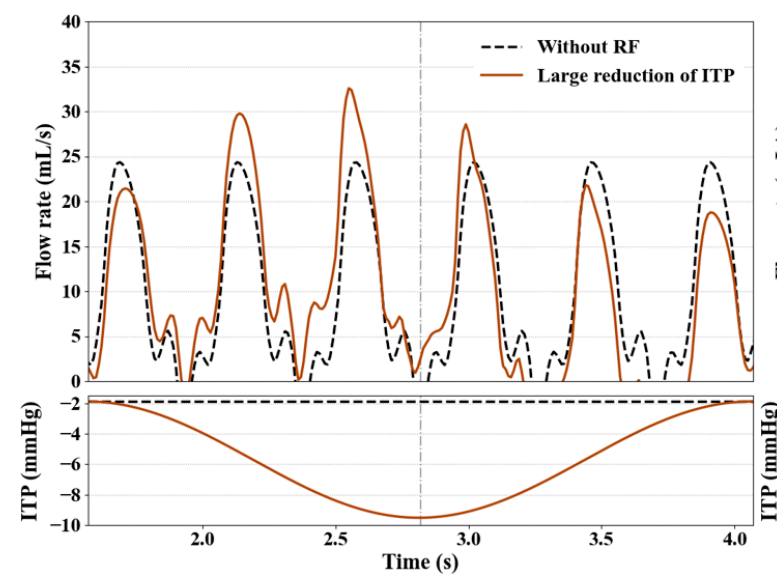

(a)

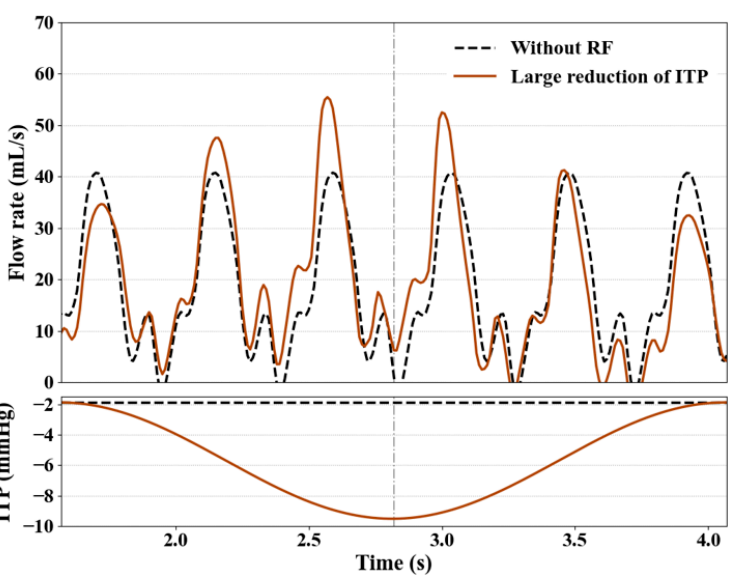

(b)

Figure 10. Impact of large ITP reduction on flow rates in SVC (a) and IVC (b) associated with an infant subject.

\subsection{Limitations}

While the current computational model is verified as capable to effectively predict the RF-induced impact on blood pressure and flow rates in CVS, the following improvements need to be further made for clinical applications. This model is merely used to predict the RF-related parameters, including frequency and amplitude, of two individual subjects in some specific breathing conditions. It is worth noting that there is often a long period of monitoring and a nursing process after admission to ICU/PICU for each patient undergoing CVD surgery. Thus, it is of great importance to establish a patient/individual-specific model to accomplish fast and accurate prediction throughout the whole-time domain for various perioperative CVDs patients. Furthermore, the information related to patients' respiration was unavailable in the collected clinical data from ICU/PICU, such as the end-tidal carbon dioxide (etCO $\mathrm{CO}_{2}$ ) recordings, and so forth $[8,40]$, which is a crucial basis for judging the presence of special respiratory fluctuation in blood pressure waveforms. Moreover, the positive pressure breathing in a ventilator is normally a significant way for 
perioperative monitoring and the treatment of patients with CVDs. Necessary ventilator use recordings and patient clinical status (awaked or anesthetized) recordings will help identify the patients' respiratory pattern in terms of ventilator-assisted breathing, spontaneous breathing, or a combination of the two. We will work with our collaborators to collect and have these data involved in our future work. In addition, the respiratory pattern can affect not only the CVS function but also lead to adaption in the nervous system, particularly in terms of the activity of autonomic nerves, which will in turn alter the CVS hemodynamics. As a key future task, we will further work on the establishment of a multi-system model integrating the CVD, RS and neural system so as to provide an effective and useful tool for clinical practice.

\section{Conclusions}

We have developed a computational model that integrates an RF model with an inhouse 0-1D multiscale hemodynamic model through adjusting ITP based on measurements, which is verified as capable to reasonably predict the RF impact on blood pressure and flow rates in both 12-year-old and 1-year-old patients. A simulation-based investigation on the impact of RF on venous return indicates that an ITP reduction in normal and deep respiratory patterns enable an increase of the blood flow rates in both SVC and IVC, while a deep breathing exercise can bring a remarkable improvement in venous return for adolescent or adult individuals but a marginal impact on infants. Moreover, a pathologically large reduction in ITP leads to excessively increasing the flow rates in SVC and IVC but independent of aging, which may enhance the hemodynamic instability and hence increase the risk of heart failure. Our results point to the potential and significance of the present 0-1D multiscale CVS model with the RF effect incorporated as an effective and useful tool for exploring the path-physiological mechanisms in association with cardiopulmonary interactions and their clinical applications.

Author Contributions: Conceptualization, K.S. and H.L.; methodology, X.Z. and R.L.; software, R.L., S.W. and Y.H.; validation, R.L.; investigation, K.S.; data curation, K.S.; writing—original draft preparation, R.L.; writing - review and editing, H.L. and K.S.; visualization, R.L.; supervision, H.L.; project administration, H.L.; funding acquisition, H.L. All authors have read and agreed to the published version of the manuscript.

Funding: The Murata Science Foundation in 2021 (PI: Koichi Sughimoto); Setsuro Fujii Memorial-The Osaka Research Foundation for Promotion of Fundamental Medical Research in 2020 (PI: Hao Liu).

Data Availability Statement: This study was approved by the Ethics Committee of Chiba Kaihin Municipal Hospital. And also, we applied Opt-out method to obtain consent on this study. The authors assert that all procedures contributing to this work comply with the ethical standards of the relevant national guidelines on human experimentation (Ethical Guidelines for Medical and Health Research Involving Human Subjects, Ministry of Health, Labor and Welfare in 2003) and with the Helsinki Declaration of 1975, as revised in 2008.

Acknowledgments: The authors acknowledge the technical supports from Chiba Kaihin Municipal Hospital for data collection. In order to assess the characteristics and shape of the arterial waveform, a newly developed novel software technology developed commercially (Medical Try Systems, Tokyo, Japan) was used to reproduce the arterial waveform.

Conflicts of Interest: The authors declare no conflict of interest.

\section{References}

1. Dornhorst, A.C.; Howard, P.; Leathart, G.L. Respiratory variations in blood pressure. Circulation 1952, 6, 553-558. [CrossRef] [PubMed]

2. Brecher, G.A.; Hubay, C.A. Pulmonary blood flow and venous return during spontaneous respiration. Circ. Res. 1955, 3, 210-214. [CrossRef] [PubMed]

3. Moreno, A.H.; Burchell, A.R.; Van der Woude, R.; Burke, J.H. Respiratory regulation of splanchnic and systemic venous return. Am. J. Physiol.-Leg. Content 1967, 213, 455-465. [CrossRef] 
4. Robotham, J.L.; Lixfeld, W.; Holland, L.; MacGregor, D.; Bryan, A.C.; Rabson, J. Effects of respiration on cardiac performance. J. Appl. Physiol. 1978, 44, 703-709. [CrossRef]

5. Kim, B.H.; Ishida, Y.; Tsuneoka, Y.; Matsubara, N.; Hiraoka, T.; Takeda, H.; Inoue, M.; Kamada, T.; Kimura, K.; Kozuka, T Effects of spontaneous respiration on right and left ventricular function: Evaluation by respiratory and ECG gated radionuclide ventriculography. J. Nucl. Med. 1987, 28, 173-177.

6. Peters, J.; Fraser, C.; Stuart, R.S.; Baumgartner, W.; Robotham, J.L. Negative intrathoracic pressure decreases independently left ventricular filling and emptying. Am. J. Physiol.-Heart Circ. Physiol. 1989, 257, H120.

7. Innes, J.A.; De Cort, S.C.; Kox, W.; Guz, A. Within-breath modulation of left ventricular function during normal breathing and positive-pressure ventilation in man. J. Physiol. 1993, 460, 487-502. [CrossRef]

8. Convertino, V.A. Mechanisms of inspiration that modulate cardiovascular control: The other side of breathing. J. Appl. Physiol. 2019, 127, 1187-1196. [CrossRef]

9. Liu, H.; Liang, F.; Wong, J.; Fujiwara, T.; Ye, W.; Tsubota, K.I.; Sugawara, M. Multi-scale modeling of hemodynamics in the cardiovascular system. Acta Mech. Sin. 2015, 31, 446-464. [CrossRef]

10. Avolio, A.P. Multi-branched model of the human arterial system. Med. Biol. Eng. Comput. 1980, 18, 709-718. [CrossRef]

11. Taylor, C.A.; Figueroa, C.A. Patient-specific modeling of cardiovascular mechanics. Annu. Rev. Biomed. Eng. 2009, 11, 109-134 [CrossRef] [PubMed]

12. Mynard, J.P.; Smolich, J.J. One-dimensional haemodynamic modeling and wave dynamics in the entire adult circulation. Ann. Biomed. Eng. 2015, 43, 1443-1460. [CrossRef] [PubMed]

13. Sherwin, S.J.; Formaggia, L.; Peiro, J.; Franke, V. Computational modelling of 1D blood flow with variable mechanical properties and its application to the simulation of wave propagation in the human arterial system. Int. J. Numer. Methods Fluids 2003, 43, 673-700. [CrossRef]

14. Sherwin, S.J.; Franke, V.; Peiró, J.; Parker, K. One-dimensional modelling of a vascular network in space-time variables. J. Eng. Math. 2003, 47, 217-250. [CrossRef]

15. Van de Vosse, F.N.; Stergiopulos, N. Pulse wave propagation in the arterial tree. Annu. Rev. Fluid Mech. 2011, 43, 467-499. [CrossRef]

16. Shi, Y.; Lawford, P.; Hose, R. Review of zero-D and 1-D models of blood flow in the cardiovascular system. Biomed. Eng. Online 2011, 10, 1-38. [CrossRef]

17. Westerhof, N.; Lankhaar, J.W.; Westerhof, B.E. The arterial windkessel. Med. Biol. Eng. Comput. 2009, 47, 131-141. [CrossRef]

18. Formaggia, L.; Lamponi, D.; Tuveri, M.; Veneziani, A. Numerical modeling of 1D arterial networks coupled with a lumped parameters description of the heart. Comput. Methods Biomech. Biomed. Eng. 2006, 9, 273-288. [CrossRef]

19. Liang, F.Y.; Takagi, S.; Himeno, R.; Liu, H. Biomechanical characterization of ventricular-arterial coupling during aging: A multi-scale model study. J. Biomech. 2009, 42, 692-704. [CrossRef]

20. Liang, F.Y.; Takagi, S.; Himeno, R.; Liu, H. Multi-scale modeling of the human cardiovascular system with applications to aortic valvular and arterial stenoses. Med. Biol. Eng. Comput. 2009, 47, 743-755. [CrossRef]

21. Liang, F.Y.; Liu, H.; Takagi, S. The effects of brachial arterial stiffening on the accuracy of oscillometric blood pressure measurement: A computational model study. J. Biomech. Sci. Eng. 2012, 7, 15-30. [CrossRef]

22. Liang, F.Y.; Takagi, S.; Liu, H. The influences of cardiovascular properties on suprasystolic brachial cuff wave studied by a simple arterial-tree model. J. Mech. Med. Biol. 2012, 12, 1250040. [CrossRef]

23. Liang, F.Y.; Takagi, S.; Himeno, R.; Liu, H. A computational model of the cardiovascular system coupled with an upper-arm oscillometric cuff and its application to studying the suprasystolic cuff oscillation wave, concerning its value in assessing arterial stiffness. Comput. Methods Biomech. Biomed. Eng. 2013, 16, 141-157. [CrossRef] [PubMed]

24. Liang, F.Y.; Fukasaku, K.; Liu, H.; Takagi, S. A computational model study of the influence of the anatomy of the circle of Willis on cerebral hyperperfusion following carotid artery surgery. Biomed. Eng. Online 2011, 10, 1-22. [CrossRef] [PubMed]

25. Liang, F.Y.; Oshima, M.; Huang, H.X.; Liu, H.; Takagi, S. Numerical study of cerebroarterial hemodynamic changes following carotid artery operation: A comparison between multiscale modeling and stand-alone three-dimensional modeling. J. Biomech. Eng. 2015, 137, 101011. [CrossRef] [PubMed]

26. Liang, F.Y.; Senzaki, H.; Yin, Z.F.; Fan, Y.Q.; Sughimoto, K.; Liu, H. Transient hemodynamic changes upon changing a BCPA into a TCPC in staged Fontan operation: A computational model study. Sci. World J. 2013, 2013, 486815. [CrossRef] [PubMed]

27. Liang, F.Y.; Senzaki, H.; Kurishima, C.; Sughimoto, K.; Inuzuka, R.; Liu, H. Hemodynamic performance of the Fontan circulation compared with a normal biventricular circulation: A computational model study. Am. J. Physiol.-Heart Circ. Physiol. 2014, 307, H1056-H1072. [CrossRef]

28. Müller, L.O.; Toro, E.F. A global multiscale mathematical model for the human circulation with emphasis on the venous system. Int. J. Numer. Methods Biomed. Eng. 2014, 30, 681-725. [CrossRef] [PubMed]

29. Zhang, X.C.; Noda, S.; Himeno, R.; Liu, H. Gravitational effects on global hemodynamics in different postures: A closed-loop multiscale mathematical analysis. Acta Mech. Sin. 2017, 33, 595-618. [CrossRef]

30. Zhang, X.C.; Haneishi, H.; Liu, H. Multiscale modeling of the cardiovascular system for infants, children, and adolescents: Age-related alterations in cardiovascular parameters and hemodynamics. Comput. Biol. Med. 2019, 108, 200-212. [CrossRef]

31. West, G.B.; Brown, J.H.; Enquist, B.J. A general model for the origin of allometric scaling laws in biology. Science 1997, 276, 122-126. [CrossRef] 
32. Pennati, G.; Fumero, R. Scaling approach to study the changes through the gestation of human fetal cardiac and circulatory behaviors. Ann. Biomed. Eng. 2000, 28, 442-452. [CrossRef] [PubMed]

33. Alastruey Arimon, J. Numerical Modelling of Pulse Wave Propagation in the Cardiovascular System: Development, Validation and Clinical Applications. Ph.D. Thesis, Imperial College London, London, UK, 2006.

34. Armstrong, M.K.; Schultz, M.G.; Picone, D.S.; Black, J.A.; Dwyer, N.; Roberts-Thomson, P.; Sharman, J.E. Brachial and radial systolic blood pressure are not the same: Evidence to support the popeye phenomenon. Hypertension 2019, 73, 1036-1041. [CrossRef]

35. Salim, M.A.; DiSessa, T.G.; Arheart, K.L.; Alpert, B.S. Contribution of superior vena caval flow to total cardiac output in children: A Doppler echocardiographic study. Circulation 1995, 92, 1860-1865. [CrossRef] [PubMed]

36. Kimura, B.J.; Dalugdugan, R.; Gilcrease III, G.W.; Phan, J.N.; Showalter, B.K.; Wolfson, T. The effect of breathing manner on inferior vena caval diameter. Eur. J. Echocardiogr. 2011, 12, 120-123. [CrossRef] [PubMed]

37. Natori, H.; Tamaki, S.; Kira, S. Ultrasonographic evaluation of ventilatory effect on inferior vena caval configuration. Am. Rev. Respir. Dis. 1979, 120, 421-427.

38. Cheyne, W.S.; Gelinas, J.C.; Eves, N.D. The haemodynamic response to incremental increases in negative intrathoracic pressure in healthy humans. Exp. Physiol. 2018, 103, 581-589. [CrossRef]

39. Singh, I.; Pinsky, M.R. Heart-lung interactions. In Mechanical Ventilation, 1st ed.; Papadakos, P.J., Lachmann, B., Visser-Isles, L., Eds.; W.B. Saunders: Philadelphia, PA, USA, 2007; pp. 173-184.

40. Ryan, K.L.; Cooke, W.H.; Rickards, C.A.; Lurie, K.G.; Convertino, V.A. Breathing through an inspiratory threshold device improves stroke volume during central hypovolemia in humans. J. Appl. Physiol. 2008, 104, 1402-1409. [CrossRef] 